\title{
Os efeitos do uso do ômega 3 no tratamento de pacientes com Alzheimer
}

\author{
The effects of using omega 3 in the treatment of patients with Alzheimer \\ Los efectos del uso de omega 3 en el tratamiento de pacientes con Alzheimer
}

Recebido: 01/11/2021 | Revisado: 09/11/2021 | Aceito: 16/11/2021 | Publicado: 18/11/2021

Poliane Josefa Costa

ORCID: https://orcid.org/0000-0001-9120-2019 Centro Universitário do Vale do Ipojuca, Brasil E-mail: polianecosta1@hotmail.com

Maria Micaele da Silva

ORCID: https://orcid.org/0000-0002-5263-1678

Centro Universitário do Vale do Ipojuca, Brasil

E-mail: micaelesilvaewellyn123@gmail.com

João Paulo Guedes

ORCID: https://orcid.org/0000-0003-2619-5080

Centro Universitário do Vale do Ipojuca, Brasil E-mail: joao.guedes@unifavip.edu.br

\begin{abstract}
Resumo
Introdução: O processo de envelhecimento é um fator de risco para doenças neurológicas, como a doença de Alzheimer. O ômega 3 é um ácido graxo presente nas membranas neuronais e desempenha um papel fundamental no metabolismo do tecido nervoso. Objetivo: descrever os efeitos do uso do ômega 3 no tratamento de pacientes com Doença de Alzheimer. Método: trata-se de uma revisão integrativa desenvolvida nas bases de dados da LILACS, MEDLINE E EMBASE por meio dos descritores: Alzheimer Disease, Omega-3 Fatty Acids, Therapeutics e Disease Prevention . Foram localizados 483 artigos, mas 476 não atenderam aos critérios de elegibilidade e foram excluídos. Realizou-se uma análise comparativa dos dados extraídos dos artigos selecionados para a amostra final. Resultados: A amostra foi composta por sete artigos. Houve prevalência do uso de cápsulas contendo $800 \mathrm{mg}$ de ácido docosa-hexaenóico e 225 mg de ácido eicosapentaenóico. Os ácidos graxos demonstraram ter potencial de reduzir o acúmulo de proteínas beta amilóide em regiões corticais do cérebro ou até a prevenção de formação, além de aumentar a espessura cortical. Conclusão: A suplementação com ômega 3 possui efeitos benéficos no tratamento de pacientes com alzheimer ao possuir características neuroprotetoras no combate a alterações morfofisiológicas que normalmente ocorrem no ambiente patológico.
\end{abstract}

Palavras-chave: Doença de Alzheimer; Ácidos graxos ômega-3; Terapêutica.

\begin{abstract}
Introduction: The aging process is a risk factor for neurological diseases such as Alzheimer's disease. Omega 3 is a fatty acid present in neuronal membranes and plays a fundamental role in the metabolism of nervous tissue. Objective: to describe the effects of using omega 3 in the treatment of patients with Alzheimer's Disease. Method: this is an integrative review developed in LILACS, MEDLINE AND EMBASE databases using the descriptors: Alzheimer Disease, Omega-3 Fatty Acids, Therapeutics and Disease Prevention. 483 articles were found, but 476 did not meet the eligibility criteria and were excluded. A comparative analysis of the data extracted from the articles selected for the final sample was carried out. Results: The sample consisted of seven articles. There was a prevalence of the use of capsules containing $800 \mathrm{mg}$ of docosahexaenoic acid and $225 \mathrm{mg}$ of eicosapentaenoic acid. Fatty acids have been shown to have the potential to reduce the accumulation of beta amyloid proteins in cortical regions of the brain or even prevent formation, in addition to increasing cortical thickness. Conclusion: Supplementation with omega 3 has beneficial effects in the treatment of patients with Alzheimer's, as it has neuroprotective characteristics in combating morphophysiological changes that normally occur in the pathological environment.
\end{abstract}

Keywords: Alzheimer disease; Omega-3 fatty acids; Therapeutics.

\section{Resumen}

Introducción: El proceso de envejecimiento es un factor de riesgo de enfermedades neurológicas como la enfermedad de Alzheimer. El Omega 3 es un ácido graso presente en las membranas neuronales y juega un papel fundamental en el metabolismo del tejido nervioso. Objetivo: describir los efectos del uso de omega 3 en el tratamiento de pacientes con enfermedad de Alzheimer. Método: se trata de una revisión integradora desarrollada en las bases de datos LILACS, MEDLINE Y EMBASE utilizando los descriptores: Enfermedad de Alzheimer, Ácidos Grasos Omega-3, Terapéutica y Prevención de Enfermedades. Se encontraron 483 artículos, pero 476 no cumplieron con los criterios de elegibilidad y fueron excluidos. Se realizó un análisis comparativo de los datos extraídos de los artículos seleccionados para la muestra final. Resultados: La muestra estuvo conformada por siete artículos. Prevaleció el uso de cápsulas que 
contenían $800 \mathrm{mg}$ de ácido docosahexaenoico y $225 \mathrm{mg}$ de ácido eicosapentaenoico. Se ha demostrado que los ácidos grasos tienen el potencial de reducir la acumulación de proteínas beta amiloides en las regiones corticales del cerebro o incluso prevenir la formación, además de aumentar el grosor cortical. Conclusión: La suplementación con omega 3 tiene efectos beneficiosos en el tratamiento de pacientes con Alzheimer, ya que posee características neuroprotectoras para combatir los cambios morfofisiológicos que normalmente ocurren en el entorno patológico.

Palabras clave: Enfermedad de Alzheimer; Ácidos grasos omega-3; Terapéutica.

\section{Introdução}

O processo de envelhecimento é um fator de risco para o desenvolvimento de doenças crônicas como doenças cardiovasculares, tumores e doenças neurodegenerativas como a Doença de Alzheimer (Tchkonia \& Kirkland, 2018). A Doença de Alzheimer (DA) é considerada uma condição neurológica crônica degenerativa caracterizada pela perda progressiva da capacidade cognitiva do indivíduo, o que leva, eventualmente, ao seu declínio funcional (Alzheimer’s Association, 2019).

A etiologia da DA é complexa e ainda não foi totalmente elucidada, no entanto acredita-se que a patogênese desta doença esteja relacionada ao acúmulo de fragmentos de proteínas beta-amilóide, que se agregam dando origem a placas localizadas próximas a neurônios e que possuem potencial neurotóxico uma vez que causam estresse oxidativo e induzem apoptose celular (Abeysinghe, Deshapriya \& Udawatte, 2020).

A destruição ou dano causado a essas células nervosas compromete regiões responsáveis pelo processamento de informações, aprendizagem e memória, causando os sintomas de declínio cognitivo característicos da doença. Por se tratar de uma doença degenerativa progressiva, os danos ao tecido nervoso e, consequentemente, os sintomas, tendem a aumentar com o tempo ao ponto de interferir negativamente na capacidade que o indivíduo possui de realizar atividades cotidianas (Alzheimer's Association, 2019).

Por este motivo, a DA é responsável por cerca de 50-70\% dos casos de demência na população, sendo esta condição caracterizada pela perda de memória e capacidade cognitiva (Winblad et al., 2016). A DA apresenta grande relevância epidemiológica diante da constante transição demográfica da população, a qual atinge idades cada vez mais avançadas, com uma estimativa de que em 2030 existam 65,7 milhões de pessoas no mundo com esta doença e em 2050 este número deve atingir 115,4 milhões (Alzheimer's Disease International, 2019).

Os medicamentos para tratamento da DA licenciados pela anvisa e disponíveis no mercado são inibidores da acetilcolinesterase e atuam inibindo a degradação da acetilcolina, aumentando a disponibilidade deste neurotransmissor para comunicação entre as células nervosas, melhorando a função cognitiva e resultando no avanço do tratamento da DA (MedeirosFilho, Nascimento, Santos \& Frazão, 2020).

Apesar dos resultados positivos observados, o uso de inibidores da acetilcolinesterase apresenta efeitos adversos, tais como náuseas, tremores, fadiga, mialgia, vômitos e diminuição de peso, o que compromete a qualidade de vida dos pacientes que fazem uso dessa classe de medicamentos (Anand, Gill \& Mahdi, 2014).

Outro recurso terapêutico que pode ser utilizado no paciente com DA é o Ômega 3 (Chaudhary et al., 2021). Os ácidos graxos poliinsaturados de cadeia longa são pertencentes às séries ômega 3, 6 ou 9, podendo ser encontrado em dietas ricas em óleos de peixe, algas ou sementes de linho, nozes e chia (Ajith, 2018; Sozańska \& Sikorska-Szaflik, 2021).

Durante o processo de envelhecimento fisiológico existe uma tendência dos ácidos graxos presentes no tecido nervoso esgotarem, o que leva a necessidade destes serem recuperados ou suplementados com ácido docosa-hexaenóico (DHA) (Chaudhary et al., 2021). Este é um ácido do tipo ômega 3 é abundantemente presente nas membranas celulares dos neurônios (Zhou et al., 2018).

O DHA possui características neuroprotetoras e está relacionado com a manutenção do metabolismo lipídico cerebral, transdução de sinal e mecanismos de neurotransmissão, além de reduzir metabólitos do ácido araquidônico e estresse oxidativo, 
portanto, desempenhando um papel importante na prevenção da DA (Chaudhary et al., 2021).

Considerando a tendência do envelhecimento populacional no país e no mundo, e que este é um fator de risco para o desenvolvimento de doenças crônicas neurodegenerativas, como a doença de Alzheimer, estudos relacionados a esta temática e que objetivam avaliar alternativas terapêuticas para este problema de saúde pública são de grande relevância. Diante disso, elaborou-se a seguinte pergunta norteadora: "Há evidências clínicas sobre os efeitos do uso do ômega 3 na melhora do tratamento de pacientes com Doença de Alzheimer?” Portanto, este estudo objetivou descrever os efeitos do uso do ômega 3 no tratamento de pacientes com Doença de Alzheimer.

\section{Metodologia}

Trata-se de uma pesquisa bibliográfica do tipo revisão integrativa (RI). Estudos de RI são utilizados com o objetivo de realizar síntese de evidências sobre um determinado assunto a partir da busca por artigos primários, resultando na construção de conhecimentos capazes de responder um problema particular de pesquisa (Whittemore \& Knalf, 2005). Esta RI seguiu as etapas de: elaboração da pergunta norteadora, busca nas bases de dados, coleta de dados, análise dos artigos incluídos na amostra, discussão dos resultados e apresentação da revisão (Souza, Silva \& Carvalho, 2010).

A pergunta norteadora para condução desta pesquisa foi elaborada seguindo a estratégia PICOT (Stillwell, FineoutOverholt \& Williamson, 2010). Para construção da pesquisa, utilizou-se as seguintes bases de dados: Literatura LatinoAmericana e do Caribe em Ciências da Saúde (LILACS), Medical Literature Analysis and Retrieval System Online (MEDLINE), ambas acessadas via Biblioteca Virtual em Saúde (BVS), além da base Excerpta Medica (EMBASE). Foram utilizados os Descritores em Ciência da Saúde (DeCS) nos idiomas português, inglês e espanhol para busca de artigos: "Doença de Alzheimer/Alzheimer Disease/Enfermedad de Alzheimer", "Ácidos Graxos Ômega-3/Omega-3 Fatty Acids/Ácidos Grasos Omega-3", "Terapêutica/Therapeutics/Terapéutica" e "Prevenção de Doenças/Disease Prevention/Prevención de Enfermedades". O operador booleano "AND” foi utilizado para combinar os descritores mencionados, conforme apresentado no Quadro a 1.

Quadro 1. Estratégia de busca nas bases de dados.

\begin{tabular}{|c|c|c|}
\hline Combinação de descritores e operador booleano & Bases & Artigos localizados \\
\hline $\begin{array}{l}\text { (doença de alzheimer) AND (ácidos graxos ômega-3) AND } \\
\text { (terapêutica) }\end{array}$ & $\begin{array}{ll} & \text { MEDLINE } \\
\text { - } & \text { LILACS }\end{array}$ & 2 \\
\hline $\begin{array}{l}\text { (Doença de Alzheimer) AND (Ácidos Graxos Ômega-3) } \\
\text { AND (Prevenção de Doenças) }\end{array}$ & $\begin{array}{ll}\text { - } & \text { MEDLINE } \\
\text { - } & \text { LILACS }\end{array}$ & 74 \\
\hline $\begin{array}{l}\text { (Alzheimer Disease) AND (Omega-3 Fatty Acids) AND } \\
\text { (Therapeutics) }\end{array}$ & $\begin{array}{ll}\text { - } & \text { MEDLINE } \\
\text { - } & \text { LILACS }\end{array}$ & 3 \\
\hline $\begin{array}{l}\text { (Alzheimer Disease) AND (Omega-3 Fatty Acids) AND } \\
\text { (Disease Prevention) }\end{array}$ & $\begin{array}{ll}\text { - } & \text { MEDLINE } \\
\text { - } & \text { LILACS } \\
\text { - } & \text { EMBASE }\end{array}$ & 324 \\
\hline $\begin{array}{l}\text { (Enfermedad de Alzheimer) AND (Ácidos Grasos Omega- } \\
\text { 3) AND (Terapéutica) }\end{array}$ & $\begin{array}{ll}\text { - } & \text { MEDLINE } \\
\text { - } & \text { LILACS }\end{array}$ & 2 \\
\hline $\begin{array}{l}\text { (Enfermedad de Alzheimer) AND (Ácidos Grasos Omega- } \\
\text { 3) AND (Prevención de Enfermedades) }\end{array}$ & $\begin{array}{ll}\text { - } & \text { MEDLINE } \\
\text { - } & \text { LILACS }\end{array}$ & 78 \\
\hline
\end{tabular}

Fonte: Autores (2021). 
A seleção de estudos foi realizada por dois pesquisadores de maneira independente. Em seguida, os resultados dos dois pesquisadores foram comparados e, em caso de divergência de seleção de estudos, foi realizado um debate para entrar em um consenso sobre a decisão final para seleção ou exclusão do estudo. Foram incluídos disponíveis gratuitamente para leitura, que fossem do tipo estudo clínico, nos idiomas inglês, espanhol ou português e que abordassem a utilização do ômega 3 no tratamento ou prevenção da doença de Alzheimer em pacientes. Os critérios de exclusão foram artigos duplicatas, cartas ao leitor e artigos de revisão. Os artigos que atenderam aos critérios de elegibilidade foram analisados por dois pesquisadores independentes no que tange ao delineamento metodológico, a fim de garantir estudos do tipo clínico que pudessem contribuir para responder a pergunta norteadora desta revisão. Os artigos estavam passíveis de exclusão no momento da análise de qualidade.

Os pesquisadores utilizaram um instrumento de coleta para extração de dados dos artigos da amostra final com as seguintes variáveis de interesse: artigos (título, país, autores, ano de publicação, revista e idioma), assim como questões que remetem a metodologia utilizada pelos artigos da amostra final (tipo de estudo e amostra/população) e questões sobre os efeitos observados a partir do uso do ômega 3 (dosagem, via de administração, ação terapêutica, efeitos colaterais, redução de sintomas). Os dados coletados foram dispostos em uma planilha e analisados a partir da leitura exaustiva do material, os quais serão categorizados e agrupados para facilitar a análise. Por fim, para apresentar os resultados da RI, utilizou-se um quadro.

\section{Resultados}

Foram localizados 483 artigos nas bases de dados, no entanto 162 eram duplicatas e, portanto, foram excluídos. Em seguida, 314 artigos foram excluídos por não atenderem aos critérios de inclusão e exclusão. Desse modo, sete artigos seguiram para a etapa de elegibilidade, onde todos foram lidos na íntegra, na qual nenhum artigo foi excluído, conforme apresentado na Figura 1.

Figura 1. Fluxograma de seleção de estudos para a amostra final.

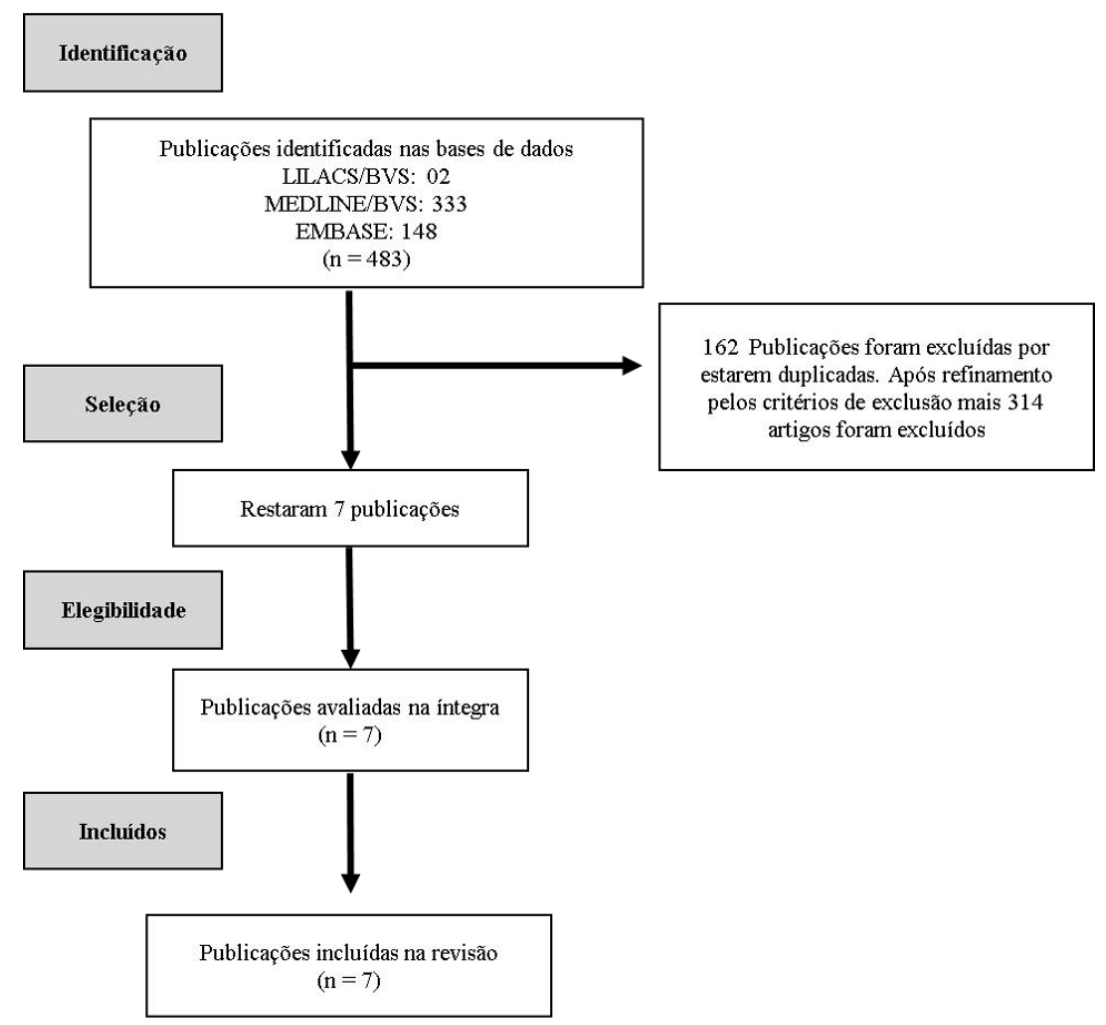

Fonte: Autores (2021). 
Sete artigos foram selecionados para a amostra final. Todos os artigos estavam na língua inglesa e foram produzidos por pesquisadores de diferentes países, principalmente da França (Andrieu et al., 2017; Giudici et al., 2020; Hooper et al., 2020), mas também da Espanha (Sala-Vila et al., 2021), Alemanha (Köbe et al., 2016), Berlim (Schwarz et al., 2018) e Estados Unidos (Fiala et al., 2020).

No que diz respeito às questões metodológicas, observou-se prevalência de ensaios clínicos randomizados (Andrieu $e t$ al., 2017; Schwarz et al., 2018; Giudici et al., 2020; Hooper et al., 2020), além de estudos clínicos (Köbe et al., 2016; Fiala et al., 2020; Sala-Vila et al., 2021). Com relação aos participantes das pesquisas apresentadas pelos artigos da amostra final, verificou-se que, em sua totalidade, eram idosos e predominantemente tinham comprometimento cognitivo, seja leve (Köbe $e t$ al., 2016; Andrieu et al., 2017; Schwarz et al., 2018; Fiala et al., 2020; Hooper et al., 2020) ou mais grave (Fiala et al., 2020; Hooper et al., 2020).

A maior parte da ingestão via oral de Ômega 3 pelos participantes dos estudos foi através de cápsulas contendo 800 mg de ácido docosa-hexaenóico (DHA) (Köbe et al., 2016.; Andrieu et al., 2017; Schwarz et al., 2018; Giudici et al., 2020; Hooper et al., 2020) e $225 \mathrm{mg}$ de ácido eicosapentaenóico (EPA) (Andrieu et al., 2017; Hooper et al., 2020; Giudici et al., 2020). Embora outras dosagens de DHA foram utilizadas, como $1000 \mathrm{mg}$ (Fiala et al., 2020), assim como dosagens de EPA, como $1000 \mathrm{mg}$ (Fiala et al., 2020) e $1320 \mathrm{mg}$ (Köbe et al., 2016; Schwarz et al., 2018).

Ademais, em associação as intervenções terapêuticas com suplementação de ômega 3, verificou-se nos artigos da amostra a presença de outros suplementos como outros lipídeos derivados de peixe (FIALA et al., 2020) e vitamina E (Schwarz et al., 2018). Intervenções não farmacológicas também foram implementadas junto com o ômega 3, como intervenções de múltiplos domínios (aconselhamento de atividade física, treinamento cognitivo e aconselhamento nutricional) (Andrieu et al., 2017; Hooper et al., 2020; Giudici et al., 2020). O Quadro 1 apresenta uma síntese dos dados extraídos nos artigos da amostra final.

Quadro 2. Caracterização dos artigos da amostra final.

\begin{tabular}{|c|c|c|c|c|c|}
\hline Citação & Tipo de Estudo & $\begin{array}{c}\text { Característica dos } \\
\text { Pacientes }\end{array}$ & $\begin{array}{c}\text { Dosagem de } \\
\text { ômega } 3\end{array}$ & Ação terapêutica & Principal desfecho \\
\hline $\begin{array}{l}\text { Giudici et } \\
\text { al., } 2020\end{array}$ & $\begin{array}{l}\text { Ensaio clínico } \\
\text { controlado } \\
\text { randomizado }\end{array}$ & $\begin{array}{c}\text { Indivíduos com idade } \\
\text { média de 75,3 anos } \\
\text { randomizados para um } \\
\text { grupo de intervenção de } \\
\text { múltiplos domínios mais } \\
\text { suplementação de ômega } 3\end{array}$ & $\begin{array}{l}\text { Cápsulas de } 800 \mathrm{mg} \text { de } \\
\text { ácido docosa-hexaenóico } \\
\text { e } 225 \mathrm{mg} \text { de ácido } \\
\text { eicosapentaenoico por dia }\end{array}$ & $\begin{array}{l}\text { Os dados do ensaio não } \\
\text { foram capazes de } \\
\text { intervenção no escore } \\
\text { dos níveis de capacidade } \\
\text { intrínseca. }\end{array}$ & $\begin{array}{c}\text { Houve diminuição entre } \\
\text { todos os grupos quando o } \\
\text { tempo foi considerado } \\
\text { contínuo, embora não } \\
\text { houvesse diferença } \\
\text { significativa entre os } \\
\text { grupos com placebo e } \\
\text { uso de suplementação ou } \\
\text { intervenção de múltiplos } \\
\text { domínios. }\end{array}$ \\
\hline $\begin{array}{l}\text { Hooper } \text { et } \\
\text { al., } 2020\end{array}$ & $\begin{array}{c}\text { Clínico } \\
\text { randomizado }\end{array}$ & \begin{tabular}{|c|} 
Pacientes $\geq 70$ anos e que \\
preenchiam pelo menos \\
um dos seguintes critérios: \\
queixas de memória \\
espontânea, limitação na \\
execução de $\geq 1$ atividade \\
instrumental da vida diária \\
ou velocidade de marcha \\
lenta $(<0,8$ metros $/ \mathrm{s})$
\end{tabular} & $\begin{array}{c}\text { Cápsulas de } 800 \text { mg de } \\
\text { ácido docosa-hexaenóico } \\
\text { e } 225 \mathrm{mg} \text { de ácido } \\
\text { eicosapentaenóico por } \\
\text { dia. }\end{array}$ & $\begin{array}{l}\text { Redução ou prevenção } \\
\text { do acúmulo de proteínas } \\
\text { beta amilóide cortical }\end{array}$ & $\begin{array}{c}\text { A intervenção no estilo } \\
\text { de vida, com ou sem } \\
\text { suplementação de ômega } \\
\text { 3, foi associada a menos } \\
\text { beta amilóide cortical em } \\
\text { adultos mais velhos em } \\
\text { risco de desenvolvimento } \\
\text { de demência. }\end{array}$ \\
\hline
\end{tabular}




\begin{tabular}{|c|c|c|c|c|c|}
\hline $\begin{array}{c}\text { Fiala } \text { et al., } \\
2020\end{array}$ & Clínico & $\begin{array}{c}\text { Pacientes idosos } \\
\text { neurodegenerativos que } \\
\text { não obtiveram sucesso em } \\
\text { suas terapias ou com } \\
\text { comprometimento } \\
\text { cognitivo leve ou } \\
\text { demência decorrente da } \\
\text { doença de alzheimer }\end{array}$ & \begin{tabular}{|} 
Suplementação diária de \\
Smartfish, bebida \\
composta por uma \\
emulsão de $200 \mathrm{~mL}, 1000$ \\
mg de de ácido docosa- \\
hexaenóico, 1000 mg de \\
ácido eicosapentaenóico e \\
outros lipídios derivados \\
de peixes
\end{tabular} & $\begin{array}{c}\text { Regula positivamente a } \\
\text { transcrição de genes } \\
\text { circadianos e de energia } \\
\text { em células } \\
\text { mononucleares de } \\
\text { sangue periférico e } \\
\text { macrófagos, melhora a } \\
\text { fagocitose e atrasa a } \\
\text { progressão para } \\
\text { demência em } 26 \text { a } 54 \\
\text { meses }\end{array}$ & $\begin{array}{l}\text { A terapia adicional com } \\
\text { ômega } 3 \text { pode atrasar a } \\
\text { demência em alguns } \\
\text { pacientes que não } \\
\text { obtiveram sucesso com } \\
\text { inibidores de } \\
\text { colinesterase } \\
\text { terapêuticos, utilizados } \\
\text { no tratamento de } \\
\text { Alzheimer. }\end{array}$ \\
\hline $\begin{array}{l}\text { Sala-Vila } \\
\text { et al., } 2021\end{array}$ & Clínico & $\begin{array}{c}\text { Participantes do estudo } \\
\text { ALFA (ALzheimer and } \\
\text { FAmilies) que são } \\
\text { portadores de APOE-ع4 } \\
\text { em abundância, muitos } \\
\text { deles parentes de pacientes } \\
\text { com DA, e que estavam } \\
\text { sob ingestão de ácido } \\
\text { docosa-hexaenóicoauto- } \\
\text { relatada }\end{array}$ & Não especificada & $\begin{array}{l}\text { O ácido docosa- } \\
\text { hexaenóico esteve } \\
\text { significativamente } \\
\text { relacionado à maior } \\
\text { espessura cortical na } \\
\text { assinatura da AD em } \\
\text { homozigotos, mas não } \\
\text { em não homozigotos }\end{array}$ & $\begin{array}{c}\text { A ingestão de ácido } \\
\text { docosa-hexaenóicona } \\
\text { dieta está relacionada a } \\
\text { padrões estruturais que } \\
\text { podem resultar em maior } \\
\text { resiliência à patologia da } \\
\text { DA Em homozigotos de } \\
\text { APOE - } \varepsilon 4 \text { sem } \\
\text { comprometimento } \\
\text { cognitivo }\end{array}$ \\
\hline $\begin{array}{c}\text { Schwarz et } \\
\text { al., } 2018\end{array}$ & $\begin{array}{c}\text { Randomizado } \\
\text { controlado }\end{array}$ & $\begin{array}{c}\text { Pacientes com } \\
\text { comprometimento } \\
\text { cognitivo leve, idade entre } \\
50-80 \text { anos, uma queixa } \\
\text { cognitiva subjetiva, um } \\
\text { comprometimento da } \\
\text { memória objetiva sem } \\
\text { comprometimento das } \\
\text { atividades da vida diária e } \\
\text { ausência de demência }\end{array}$ & \begin{tabular}{|c|} 
A ingestão diária de \\
quatro cápsulas \\
combinadas em um total \\
de $2200 \mathrm{mg}$ de ômega-3 \\
de cadeia longa (1320 mg \\
de ácido \\
eicosapentaenóico, 880 \\
mg de ácido docosa- \\
hexaenóico e 15 mg de \\
vitamina E adicionais)
\end{tabular} & $\begin{array}{l}\text { Aumento da perfusão } \\
\text { cerebral após } \\
\text { suplementação de } \\
\text { ômega-3 }\end{array}$ & $\begin{array}{c}\text { A suplementação de } \\
\text { ômega-3 pode beneficiar } \\
\text { regiões cerebrais afetadas } \\
\text { pelo comprometimento } \\
\text { cognitivo leve com o } \\
\text { aumento da perfusão. } \\
\text { Ademais, os resultados } \\
\text { sugerem que a } \\
\text { suplementação tem } \\
\text { potencial terapêutico, } \\
\text { podendo prevenir a } \\
\text { Doença de Alzheimer. }\end{array}$ \\
\hline $\begin{array}{c}\text { Andrieu } \text { et } \\
\text { al. }, 2017\end{array}$ & Randomizado & $\begin{array}{c}\text { Os participantes não } \\
\text { tinham demência, tinham } \\
70 \text { anos ou mais e viviam } \\
\text { na comunidade e relataram } \\
\text { queixas de memória } \\
\text { espontânea ao médico, } \\
\text { limitações em uma } \\
\text { atividade instrumental da } \\
\text { vida diária ou velocidade } \\
\text { de marcha lenta. }\end{array}$ & $\begin{array}{c}\text { Dose diária total de } \\
\text { cápsulas contendo } 800 \\
\text { mg de ácido docosa- } \\
\text { hexaenóico e } 225 \mathrm{mg} \text { de } \\
\text { ácido eicosapentaenóico }\end{array}$ & $\begin{array}{l}\text { Não houve declínio } \\
\text { cognitivo significativo } \\
\text { ao longo dos } 3 \text { anos de } \\
\text { estudo. }\end{array}$ & $\begin{array}{c}\text { A intervenção de } \\
\text { múltiplos domínios e os } \\
\text { ácidos graxos } \\
\text { poliinsaturados, sozinhos } \\
\text { ou em combinação, não } \\
\text { tiveram efeitos } \\
\text { significativos no declínio } \\
\text { cognitivo ao longo de } 3 \\
\text { anos em idosos com } \\
\text { queixas de memória. }\end{array}$ \\
\hline $\begin{array}{c}\text { Köbe } e t \\
a l ., 2016 .\end{array}$ & Clínico & $\begin{array}{c}\text { Pacientes (com idades } \\
\text { entre } 60-80 \text { anos) } \\
\text { diagnosticados com queixa } \\
\text { cognitiva subjetiva e um } \\
\text { comprometimento de } \\
\text { memória objetivo em } \\
\text { testes padronizados }\end{array}$ & $\begin{array}{c}4 \text { cápsulas contendo } 1320 \\
\text { mg de ácido } \\
\text { eicosapentaenóico, } 880 \\
\text { mg de ácido docosa- } \\
\text { hexaenóico }\end{array}$ & $\begin{array}{l}\text { Ômega-3 por si só não } \\
\text { evitou a atrofia mas a } \\
\text { combinação das } \\
\text { intervenções resultou em } \\
\text { diminuição da } \\
\text { homocisteína } \\
\text { correlacionada com o } \\
\text { aumento do volume } \\
\text { regional de matéria } \\
\text { cinzenta }\end{array}$ & $\begin{array}{l}\text { Intervenção combinada } \\
\text { com ácidos graxos } \\
\text { ômega-3, exercícios } \\
\text { aeróbicos e estimulação } \\
\text { cognitiva reduziu } \\
\text { significativamente a } \\
\text { atrofia da substância } \\
\text { cinzenta em regiões } \\
\text { cerebrais relacionadas à } \\
\text { DA em pacientes }\end{array}$ \\
\hline
\end{tabular}

Fonte: Autores (2021).

\section{Discussão}

Através da condução de uma revisão integrativa, este artigo visou descrever o uso do ômega 3 no tratamento de pacientes com Doença de Alzheimer. Os dados coletados nos artigos da amostra final permitiram identificar pesquisas em diferentes partes do mundo voltadas para avaliação de intervenções com ômega 3 em indivíduos com idade avançada com comprometimento neurológico em risco ou já acometido pela doença de Alzheimer, e em alguns casos apresentavam alterações de memória ou de 
realizar atividades de vida diária (Andrieu et al., 2017; Schwarz et al., 2018; Hooper et al., 2020; Sala-Vila et al., 2021).

No entanto, ressalta-se que não existem muitos artigos sobre a temática, inclusive nenhum dos artigos tinham procedência no Brasil. Diante disso, acredita-se identifica-se a necessidade de investimento em pesquisas que avaliem os efeitos do ômega 3, inclusive em território nacional, considerando que já existem relatos na literatura que sugerem seus efeitos benéficos para os pacientes com Alzheimer, conforme aponta os resultados desta pesquisa de revisão.

A predominância da população idosa nos estudos é um cenário já conhecido na comunidade científica, estando atrelado ao próprio processo de envelhecimento (Tchkonia \& Kirkland, 2018), por exemplo, um estudo realizado em uma capital do nordeste identificou que a prevalência da DA aumenta a partir dos 60 anos, apresentando maior prevalência para o sexo feminino $(69,6 \%)$ do que para o sexo masculino $(30,6 \%)$ (Soares et al., 2017).

De acordo com os artigos da amostra, a suplementação com ômega 3 foi feita principalmente com o ácido docosahexaenóico (DHA) (Köbe et al., 2016.; Andrieu et al., 2017; Schwarz et al., 2018; Giudici et al., 2020; Hooper et al., 2020) e 225 mg de ácido eicosapentaenóico (EPA) (Andrieu et al., 2017; Hooper et al., 2020; Giudici et al., 2020). Trata-se de um ácido graxo presente nas membranas neuronais que desempenha função neuroprotetora, além de auxiliar na comunicação entre as células do tecido nervoso e reduzir metabólitos do ácido araquidônico e estresse oxidativo, justificando seu uso nas intervenções terapêuticas na Doença de Alzheimer (Chaudhary et al., 2021).

Além disso, o ácido eicosapentaenóico (EPA) também foi muito mencionado pelos artigos da amostra (Köbe et al., 2016; Andrieu et al., 2017; Schwarz et al., 2018; Hooper et al., 2020; Giudici et al., 2020). Trata-se de um ácido graxo do tipo ômega 3 que também está presente no tecido nervoso e que, além de auxiliar no metabolismo tecidual também possui potencial neurológico ao suprimir a produção e deposição de proteínas beta amilóides (ZHOU et al., 2018), sendo este um dos mecanismos patológicos da Doença de Alzheimer (Alzheimer's Association, 2019).

Diante deste contexto, a análise dos resultados permitiu identificar benefícios relacionados ao uso de ácidos graxos do tipo ômega 3, como o EPA e o DHA, os quais demonstraram ter potencial de reduzir do acúmulo de proteínas beta amilóide em regiões corticais do cérebro ou até a prevenção de formação (Hooper et al., 2020).

Estes achados corroboram com a literatura, em que um estudo realizado por $\mathrm{Xu}$ et al. (2021) sugere ainda que a suplementação de EPA e DHA enriquecidos reduzem significativamente a produção de beta amilóide em células do hipocampo, além de protegerem neurônios primários desta região contra a toxicidade induzida por proteínas beta amilóides, estimulando seu crescimento celular e sua atividade fagocítica de células nervosas.

Quanto às alterações anatômicas no cérebro, evidências científicas apontam uma possível associação entre ácidos graxos ômega 3 e a morfologia de regiões cerebrais, em que o maior nível de ômega três esteve frequentemente envolvido com maior volume no hipocampo, maior massa cinzenta total e menor lesão de substância branca (Macaron et al., 2021). Diante disso, os resultados desta revisão integrativa apontaram que a ingestão de ômega 3 aumentam a espessura cortical (Sala-Vila et al., 2021), além de que, em combinação com outras intervenções voltadas para cognição, reduziu regiões cerebrais de atrofia (Köbe et al., 2016). Portanto, a suplementação de ômega 3 pode conferir resiliência à Doença de Alzheimer.

Desse modo, destaca-se também que os efeitos terapêuticos do ômega 3 podem ser potencializados quando utilizado em conjunto com outras intervenções, como intervenções não farmacológicas apontadas pelos artigos da amostra como: aconselhamento de atividade física, treinamento cognitivo e aconselhamento nutricional (Andrieu et al., 2017; Giudici et al., 2020).

Os protocolos de tratamento farmacológicos, os pacientes podem receber intervenções não farmacológicos, que ocorrem a partir da implementação de atividades motoras que estimulam o idoso, como aeróbica, assim como a prática de atividades que estimulam a capacidade cognitiva através de leituras e jogos e também musicoterapia. Desse modo, melhorando a qualidade de vida do paciente ao reduzir a dependência que os pacientes podem possuir para executar atividades de vida diária (Costa, Dantas, 
Dantas, Oliveira, Aiquoc \& Sarmento, 2019).

\section{Conclusão}

Diante do que foi exposto, conclui-se que o ômega 3 possui potencial terapêutico para pacientes com Doença de Alzheimer, ao possuir características neuroprotetoras que estimulam o metabolismo neuronal e crescimento celular, além de auxiliar na prevenção de acúmulo de proteínas beta amiloides no tecido nervoso, que normalmente ocorre no ambiente patológico da Doença de Alzheimer. Ressalta-se ainda a combinação terapêutica de intervenções não farmacológicas que busquem auxiliar o desenvolvimento cognitivo dos pacientes com Alzheimer ou com risco para, a fim de potencializar a ação benéfica do ômega 3.

\section{Referências}

Abeysinghe, A. A. D. T., Deshapriya, R. D. U. S., \& Udawatte, C. (2020). Alzheimer's disease; a review of the pathophysiological basis and therapeutic interventions. Life Sciences, 256, 117996.

Andrieu, S., Guyonnet, S., Coley, N., Cantet, C., Bonnefoy, M., Bordes, S., ... \& Olivier-Abbal, P. (2017). Effect of long-term omega 3 polyunsaturated fatty acid supplementation with or without multidomain intervention on cognitive function in elderly adults with memory complaints (MAPT): a randomised, placebocontrolled trial. The Lancet Neurology, 16(5), 377-389.

Ajith, T. A. (2018). A recent update on the effects of omega-3 fatty acids in Alzheimer's disease. Current clinical pharmacology, 13(4), 252-260.

Alzheimer's Association. (2019). 2019 Alzheimer's disease facts and figures. Alzheimer's \& dementia, 15(3), $321-387$.

Prince, M. J., Wimo, A., Guerchet, M. M., Ali, G. C., Wu, Y. T., \& Prina, M. (2015). World Alzheimer Report 2015-The Global Impact of Dementia: An analysis of prevalence, incidence, cost and trends.

Anand, R., Gill, K. D., \& Mahdi, A. A. (2014). Therapeutics of Alzheimer's disease: Past, present and future. Neuropharmacology, 76, 27-50.

Costa, I. B., Dantas, D. V., Dantas, R. A. N., Oliveira, S. P., Aiquoc, K. M., \& Sarmento, S. D. G. (2019). Terapias não farmacológicas para o manejo da dor em vítima de queimaduras: uma revisão sistemática. Revista Inspirar: movimento e saúde, 19(2), 1-20.

Chaudhary, V., Nigam, A. K., Paliwal, A., Singh, M. K., Gour, J. K., \& Sinha, V. B. (2021). Omega 3 PUFA. In Naturally Occurring Chemicals Against Alzheimer's Disease (pp. 65-82). Academic Press.

Engelhardt, E., Brucki, S. M., Cavalcanti, J. L. S., Forlenza, O. V., Laks, J., \& Vale, F. A. (2005). Tratamento da Doença de Alzheimer: recomendações e sugestões do Departamento Científico de Neurologia Cognitiva e do Envelhecimento da Academia Brasileira de Neurologia. Arquivos de Neuro-psiquiatria, 63, 1104-1112.

Fiala, M., Lau, Y. C. C., Aghajani, A., Bhargava, S., Aminpour, E., Kaczor-Urbanowicz, K. E., ... \& Pellegrini, M. (2020). Omega-3 Fatty Acids Increase Amyloid- $\beta$ Immunity, Energy, and Circadian Rhythm for Cognitive Protection of Alzheimer's Disease Patients Beyond Cholinesterase Inhibitors. Journal of Alzheimer's Disease, 75(3), 993-1002.

Giudici, K. V., Barreto, P. S., Beard, J., Cantet, C., de Carvalho, I. A., Rolland, Y., \& Vellas, B. (2020). Effect of long-term omega-3 supplementation and a lifestyle multidomain intervention on intrinsic capacity among community-dwelling older adults: secondary analysis of a randomized, placebo-controlled trial (MAPT study). Maturitas, 141, 39-45.

Hooper, C., Coley, N., Barreto, P. D. S., Payoux, P., Salabert, A. S., Andrieu, S., ... \& Vellas, B. (2020). Cortical $\beta$-Amyloid in Older Adults Is Associated with Multidomain Interventions with and without Omega 3 Polyunsaturated Fatty Acid Supplementation. The journal of prevention of Alzheimer's disease, 7(2), 128134.

Köbe, T., Witte, A. V., Schnelle, A., Lesemann, A., Fabian, S., Tesky, V. A., ... \& Flöel, A. (2016). Combined omega-3 fatty acids, aerobic exercise and cognitive stimulation prevents decline in gray matter volume of the frontal, parietal and cingulate cortex in patients with mild cognitive impairment. Neuroimage, 131 , 226-238.

Macaron, T., Giudici, K. V., Bowman, G. L., Sinclair, A., Stephan, E., Vellas, B., \& de Souto Barreto, P. (2021). Associations of Omega-3 fatty acids with brain morphology and volume in cognitively healthy older adults: A narrative review. Ageing Research Reviews, 67, 101300.

Medeiros Filho, F. C., dos Santos Nascimento, K., Santos, W. O., \& Frazão, N. F. (2020). Estudo Da Inibição Da Acetilcolinesterase Por Docking Molecular: Aplicação No Tratamento Da Doença Do Alzheimer. Educação, Ciência E Saúde, 7(2), 18.

Pais, M., Martinez, L., Ribeiro, O., Loureiro, J., Fernandez, R., Valiengo, L., ... \& Forlenza, O. V. (2020). Early diagnosis and treatment of Alzheimer's disease: new definitions and challenges. Brazilian Journal of Psychiatry, 42, 431-441.

Sala-Vila, A., Arenaza-Urquijo, E. M., Sánchez-Benavides, G., Suárez-Calvet, M., Milà-Alomà, M., Grau-Rivera, O., ... \& ALFA study. (2021). DHA intake relates to better cerebrovascular and neurodegeneration neuroimaging phenotypes in middle-aged adults at increased genetic risk of Alzheimer disease. The American journal of clinical nutrition, 113(6), 1627-1635. 
Research, Society and Development, v. 10, n. 15, e239101522654, 2021

(CC BY 4.0) | ISSN 2525-3409 | DOI: http://dx.doi.org/10.33448/rsd-v10i15.22654

Schwarz, C., Wirth, M., Gerischer, L., Grittner, U., Witte, A. V., Köbe, T., \& Flöel, A. (2018). Effects of Omega-3 fatty acids on resting cerebral perfusion in patients with mild cognitive impairment: a randomized controlled trial. The journal of prevention of Alzheimer's disease, 5(1), 26-30.

Soares, N. M., Pereira, G. M., da Nóbrega Figueiredo, R. I., Soares, N. M., de Almeida, R. M. M., \& da Silva Portela, A. (2017). Impacto econômico e prevalência da doença de Alzheimer em uma capital Brasileira. Ciência \& Saúde, 10(3), 133-138.

Souza, M. T. D., Silva, M. D. D., \& Carvalho, R. D. (2010). Integrative review: what is it? How to do it?. Einstein (São Paulo), 8, $102-106$.

Sozańska, B., \& Sikorska-Szaflik, H. (2021). Diet Modifications in Primary Prevention of Asthma. Where Do We Stand?. Nutrients, $13(1)$, 173.

Stillwell, S. B., Fineout-Overholt, E., Melnyk, B. M., \& Williamson, K. M. (2010). Evidence-based practice, step by step: asking the clinical question: a key step in evidence-based practice. AJN The American Journal of Nursing, 110(3), 58-61.

Tchkonia, T., \& Kirkland, J. L. (2018). Aging, cell senescence, and chronic disease: emerging therapeutic strategies. Jama, 320(13), 1319-1320.

Whittemore, R., \& Knafl, K. (2005). The integrative review: updated methodology. Journal of advanced nursing, 52(5), 546-553.

Winblad, B., Amouyel, P., Andrieu, S., Ballard, C., Brayne, C., Brodaty, H., ... \& Zetterberg, H. (2016). Defeating Alzheimer's disease and other dementias: a priority for European science and society. The Lancet Neurology, 15(5), 455-532.

Xu, Z. J., Li, Q., Ding, L., Shi, H. H., Xue, C. H., Mao, X. Z., ... \& Zhang, T. T. (2021). A comparative study of the effects of phosphatidylserine rich in DHA and EPA on A $\beta$-induced Alzheimer's disease using cell models. Food \& Function, 12(10), 4411-4423.

Zhou, M. M., Che, H. X., Huang, J. Q., Zhang, T. T., Xu, J., Xue, C. H., \& Wang, Y. M. (2018). Comparative study of different polar groups of EPA-enriched phospholipids on ameliorating memory loss and cognitive deficiency in aged SAMP8 mice. Molecular nutrition \& food research, $62(7), 1700637$. 